\title{
LAS PRINCIPALES CONCEPCIONES PSICOANTROPOLÓGICAS COMO REFERENTE PARA LA ACTIVIDAD PROFESIONAL DEL PSICÓLOGO
}

\author{
Ps. Luis García Vega
}

\begin{abstract}
RESUMEN
En este artículo se presentan los lineamientos generales de 5 modelos teóricos provenientes de la Psicología que en la actualidad se utilizan para describir al hombre. Estos son los modelos: Conductista, Psicoanalista, Humanista, Materialista, Cognitivo y Cognitivo Emocional.

En todos los casos se presentan a los principales representantes, se señalan los antecedentes históricos de cada modelo y el tipo de explicación que desarrolla.

Finalmente se presenta la bibliografía específica para cada modelo.

PALABRAS CLAVE: Psicoantropología, actividad profesional, conductismo, psicoanalista, humanista, materialista, cognitivo, cognitivo emocional.
\end{abstract}

\begin{abstract}
The article the usual lineaments of 5 theoric models proceeding of the psychology what in the actuality be use for to sketch or man. It's are the models:

Conductist, Psychoanalist, Humanist, Materialist, Cognitive and Emotional Cognitive.

In alls the cases be present or the principals representantings be to signal the histories antecedents of each model and the type of describe which development.

Finally be present the specific bibliography for each model.

KEY WORDS: Psychoantropology, professional activity, conductist, psychoanalyst, humanist, materialist, cognitive, emotional cognitive.
\end{abstract}




\section{EL MODELO CONDUCTISTA}

Los psicólogos conductistas, entre los que destacan J.B. Watson (1878-1958) y B.F. Skinner (1904-), querían encontrar unos principios objetivos para controlar la conducta de los organismos vivos. Su afán era ser absolutamente fieles a las más rigurosas reglas del método científico. Esta idea fue expuesta por primera vez por Watson en "La psicología tal y como la ve el conductista" (Psychol. Rev., 20, 158-177, 1913). Esclavos de esta exigencia los psicólogos conductistas llegaron hasta sus últimas consecuencias, la eliminación del hombre del escenario de la psicología; quedando reducido, como afirma Skinner, a un simple epifenómeno, objeto tan sólo de "descripciones literarias".

El "hombre" conductista en muchos aspectos es equiparable a una máquina, porque en ambos sistemas el comportamiento puede ser predecido y controlado mediante leyes. "El hombre: una máquina", este es el título del cap. IV de la conocida obra de Skinner ciencia y conducta humana; lo mismo afirma Watson en El conductismo (Buenos Aires: Paidos, pag. 253 y siguientes).

El conductista reduce el comportamiento humano a las mismas leyes reguladoras de la conducta animal (véase, por ejemplo, Watson, El conductismo, pags. 67-68).

La postura conductista minimiza o prescinde del papel diferenciador de la herencia individual, defendiendo que, a efectos prácticos todos los hombres normales al nacer están igualmente dotados y poseen una gran flexibilidad para ser moldeados de formas muy diferentes, según los tipos de condicionamiento a los que quedan expuestos a lo largo de su vida:

"Un hombre está hecho del mismo material que los demás hombres y tiene idéntico plan arquitectónico general" (Watson, El conductismo, cap. VI). A nivel práctico se pueden ver las consecuencias de esta afirmación en un elocuente texto de Watson: "Dadnos una docena de niños sanos, bien formados y un mundo apropiado para criarlos, y garantizamos convertir a cualquiera de ellos, tomado al azar, en determinado especialista: médico, abogado, artista, jefe de comercio, pordiosero o ladrón, no importando los talentos, inclinaciones, tendencias, habilidades, vocaciones y raza de sus ascendientes (J.B. Watson, El conductismo, pags. 110-111).

Partiendo de estas premisas la conducta ya no es del hombre, y éste es simplemente un organismo de naturaleza biológica, que necesariamente reacciona a la situación estimular, según unas leyes que no dependen de él en lo más mínimo. La psicología deja pues de ser la ciencia que estudia la conciencia y la conducta del hombre, para convertirse en ciencia de la conducta sin más, que depende exclusivamente del estímulo. 
Skinner pretende explicar, predecir y controlar (provocar y modificar) la conducta de los organismos vivientes (incluyendo al hombre), partiendo del siguiente principio, que con frecuencia es usado en la pedagogía: Si reforzamos una conducta, ésta volverá a repetirse, y si no la reforzamos no se aprende. Si esto es verdad, cualquiera que con cierta habilidad (tecnología de la conducta) premie o castigue puede hacer que otro haga lo que él quiera mediante el refuerzo o su ausencia.

Skinner trabajó con muchos colaboradores durante casi 40 años en su Laboratorio y durante muchas miles de horas, y demostró al mundo que mediante este principio podía controlar la conducta de los animales, haciéndoles aprender a hacer lo que él quería. Según él, este principio es válido para explicar también la conducta del hombre, que inexorablemente se va haciendo desde la niñez por medio del condicionamiento de padres, educadores, sociedad, etc. en base al premio o al castigo.

El lector interesado en los tipos de programa de refuerzo y en las consecuencias que tiene cada uno en la Conducta, puede consultar a Skinner en Ciencia y conducta humana, pags. 128- 136 y algunos articulo del mismo autor en Registro acumulativo.

Según esta postura psicológica el hombre no puede ser libre, porque la conducta es función exclusivamente del condicionamiento. Al no ser libre no puede responder de sus actos ante los demás (responsabilidad). Tampoco, por tanto, es digno ni de premios ni de castigos, "No podemos, afirma Skinner, reconocer los méritos de una persona cuyas obras son de hecho debidas a fuerzas sobre las que no ejerce control alguno".

Creemos con el conductista que el condicionamiento es algo insustituible en la formación del hombre, pero por sí solo no puede explicar todas las conductas del hombre. Con frecuencia el hombre aprende y se comporta de un modo parecido a un autómata o a un animal, pero los procesos cognitivos y otros de índole superior intervienen modificando cualitativamente la conducta humana.

Los psicólogos conductistas se despreocupan inicialmente del hombre, y partiendo de una concepción metodológica cientificista, concluyen con su inoperancia a nivel de conducta, que es lo que a ellos les importa. 


\section{EL MODELO PSICOANALISTA}

Para Freud el hombre es un organismo cuya energía surge del "ello", la "provincia" o "instancia" más antigua del "aparato psíquico", su contenido más importante son los instintos, "causa última de toda actividad"; se definen operativamente como "exigencias somáticas planteadas a la vida psíquica" y funciona en virtud del inexorable "principio del placer", cuya característica es buscar inmediata e incondicionalmente la satisfacción, sin tener en cuenta las consecuencias futuras.

Las exigencias de la realidad van convirtiendo un sector del ello en el "yo". Según Freud "originalmente todo era ello, el yo se desarrolló del ello por la incesante influencia del mundo exterior". El yo se rige por el "principio de realidad" (busca el placer en el momento y manera más oportunos). El yo trata de resolver los conflictos entre el ello y las exigencias sociales. El superyo, heredero y continuador del "complejo de Edipo", resulta de las relaciones del niño con los padres, educadores, cultura, etc., que tienen lugar durante el prolongado periodo de "dependencia infantil" y cuya principal misión es "restringir las satisfacciones del ello".

El psicoanálisis como técnica pretende que el yo se fortalezca a costa del ello y frente al superyo, y logre un equilibrio.

De un yo débil resulta la neurosis. La práctica desaparición del yo da lugar a la psicosis.

La naturaleza del hombre en el psicoanálisis la constituyen estas tres instancias, y el cariz de sus relaciones dinámicas mutuas definen a cada hombre. El hombre, originaria y primariamente, es de naturaleza biológica (los instintos son la pieza clave de la vida del hombre) y, secundariamente, el hombre se socializa. Los instintos generan y se manifiestan en el hombre a través de estratos muy profundos de carácter irracional.

En su dinámica este modelo es homeostático y geneticista porque depende del pasado, de las primeras etapas de desarrollo infantil y sobre todo de una de ellas, la "etapa edípica".

A.Adler, C.Jung y E. Fromm, entre otros, han elaborado interesantes modelos explicativos del hombre que serán presentados dentro de este programa.

En los últimos cincuenta años se desarrolló una concepción optimista del hombre, el humanismo psicológico, que no estaba de acuerdo ni con la visión pesimista de Freud, ni con la ambientalista y determinista de los conductistas. 


\section{EL MODELO HUMANISTA}

El humanismo psicológico parte de la idea de que cada hombre es algo con cualidades propias cuyo origen no está en el condicionamiento. Entre estas cualidades se destaca la capacidad para hacerse a sí mismo libremente, aunque de hecho no la ejerza, porque los demás le obligan a ser de otra manera, mediante las sutiles técnicas de condicionamiento.

En líneas generales, esta postura es compartida por Gordon W. Allport (18971967), Carl R. Rogers (1902-) y Abraham H. Maslow (1908-1970).

Los psicólogos humanistas, en sus investigaciones y actividad clínica, parten de una concepción del hombre previa. Le dan importancia al hombre porque puede ser agente de la conducta. La conducta puede ser del hombre, que es quien recibe e interpreta los estímulos, reaccionando o no, y de una y otra manera ante ellos.

Para el conductista, el hombre cuando nace es como un papel en blanco, y lo que en ese papel va escribiendo la vida es lo que le va haciendo a cada uno ser lo que es. Para el psicólogo humanista, cada hombre desde el nacimiento posee un conjunto de cualidades sin desarrollar y una tendencia a caminar en una cierta dirección. Del grado de desarrollo de estas cualidades y del cumplimiento del destino de cada persona depende su nivel de maduración. Para llegar a esta conclusión, estos psicólogos defienden el valor positivo de la naturaleza original humana.

A nivel pedagógico son diferentes los papeles que van a desarrollar los educadores Skinnerianos y los humanistas. De la distinta concepción del hombre se sigue un diferente trato al alumno:

- $\mathrm{Si}$ aceptamos la concepción skinneriana del hombre, nosotros educadores debemos aprender, experimentar y desarrollar las técnicas de aprendizaje (condicionamiento por refuerzo) para aplicarlos adecuadamente, y así conseguir que el alumno aprenda lo que tiene que aprender para adecuarse y ser útil a la sociedad y cultura en la que se encuentra. Si no hacemos esto, el alumno se condicionará a hacer otras cosas (no estudiar, estudiar mal, etc.), dejándose llevar por otras circunstancias no controladas y, tal vez, no controlables. En pocas palabras, su papel es manipular y dirigir utilizando ciertas técnicas.

- Si partimos de la concepción humanista, nuestra labor consiste en favorecer que cada uno sea él mismo, enseñándole a luchar contra las múltiples influencias a las que está sometido (publicidad comercial y otras manipulaciones) para que de él partan las decisiones. No debemos obligar al alumno a ser o hacer algo determinado, sin tentarle creando una situación favorecedora para que él sea él mismo. Si no le "manipulo" mediante premios, castigos, amenazas, notas, etc. 
¿qué puedo hacer con aquellos que no quieran aprender mi asignatura?, ¿esperar? El educador humanista trata a cada uno según es. corno un hortelano que cultiva cada planta dándole el agua, abono y cuidado que necesita.

El punto de partida diferente (conducta u hombre) abre distintos caminos y conduce a resultados opuestos. Si partimos de la conducta, se logra una tecnología de la misma muy sugestiva y a la vez gratificante para los "cientistas" u apasionados por la objetividad científica. Si empezamos por el hombre, el camino y los resultados no se alejan mucho de la filosofía, y deben apoyarse en una sólida concepción filosófica del hombre. En este caso el saber pierde la capacidad de contrastación científica exhaustiva, pero, en su lugar, sus confines se dilatan casi sin fin y penetran en profundidades del ser humano ignoradas y despreciadas por el psicólogo objetivista .

\section{EL MODELO MATERIALISTA, DIALÉCTICO DE LA PSICOLOGÍA SOVIÉTICA}

En la psicología soviética el concepto de hombre desempeña un importante papel. Él es el sujeto de la "actividad" (categoría de la psicología soviética que viene a representar la conducta, pero concebida a la luz de la ideología marxista-leninista). El hombre es resultado de un largo proceso de transformaciones dialécticas de la materia. Lo que en este proceso constituye y caracteriza al hombre es la aparición de la conciencia, distinta cualitativamente de la psique animal. La conciencia es la propiedad del cerebro humano, la más alta organización a la que llega la materia, gracias al modo de vida social en el que se desenvuelve el hombre. La vida social comienza cuando las condiciones biológicas (animales) de vida se convierten para algunos seres progresivamente en condiciones sociales de vida; las actividades trabajo-lenguaje generan este tipo de vida que a su vez repercute y transforma dichas actividades. Engels, en su pequeña obra El papel del trabajo en la transformación de mono en hombre (1876), describe anecdóticamente esta transformación y el papel del trabajo en este proceso. Marx, en la tesis $6^{\mathrm{a}}$ "A feuerbach", enfatiza el carácter histórico y social opuesto a toda consideración metafísica (de las esencias eternas) del ser humano: "La esencia humana no es algo abstracto e immanente a cada individuo. Es, en su realidad, el conjunto de relaciones sociales". Esta última frase del texto constituye el punto de partida y el término de cualquier investigación soviética sobre la personalidad.

Para la psicología soviética, una de las principales actividades de la conciencia es la de poder representar ("reflejos") "idealmente" los acontecimientos en las líneas principales de su proceso, y a la luz de los resultados finales antes de que sucedan. Es altamente significativo un texto de Marx del Capital en el que compara la conducta de la abeja y la araña con la del arquitecto. Éste puede manipular, reelaborar y ejecutar libremente el proyecto gracias a su capacidad representativa; mientras que el animal lo 
ejecuta de una manera automática, rígida (instintiva). "Una araña, dice Marx, ejecuta operaciones semejantes a las del tejedor y una abeja avergüenza, por la construcción de sus celdillas de cera, a más de un arquitecto humano. Pero lo que ya por anticipado distingue el peor arquitecto de la abeja mejor es que el arquitecto construye la celdilla en su cabeza antes de construida con cera. Al final del proceso de trabajo sale un resultado que ya estaba presente al principio del mismo en la representación del trabajador, o sea, idealmente. No es sólo que el trabajador obre una alteración de forma de la naturaleza; es que al mismo tiempo realiza en lo natural su finalidad, la cual es conocida por él, determina como ley el modo de su hacer y tiene subordinada su voluntad". Esta posibilidad de representar lo que aún no ha sucedido le permite no sólo prever, sino también preparar, elegir, planificar y programar las acciones a la luz de sus consecuencias, pudiendo controlar, por tanto, los resultados. Uno de los principios básicos que defiende la psicología soviética es el del determinismo, entendido en el sentido de que puestos unos medios necesariamente se siguen unos resultados. Se salva la libertad del hombre cuando conoce la relación necesaria de medios y fines y puede elegir unos u otros medios. La valoración de los fines en la sociedad soviética está determinada por la ideología Marxista-Leninista. El conocimiento de los pasos a dar para alcanzar tales fines forma parte del quehacer del Partido Comunista. La sólida formación ideológica Marxista-Leninista ayuda a elegir los fines y medios adecuados. Al elegir los fines y medios adecuados el hombre soviético se hace libre, pudiendo responder socialmente ante los demás del desarrollo de la sociedad socialista.

\section{EL MODELO COGNITIVO}

La mayor parte de los psicólogos conceden una especial importancia a los procesos cognitivos. El desarrollo tan manifiesto de la cibernética, de la teoría de la información, del ordenador, les incita a adoptar el procesamiento de la información como modelo adecuado para la psicología. El estudio de los procesos cognitivos ocupa gran parte del quehacer de los psicólogos actuales.

El modelo de hombre como procesador de información ofrece una alternativa diferente a las anteriores.

Para Neisser, uno de los pioneros de esta nueva orientación en psicología, el hombre está tanto más desarrollado y es más libre si posee "esquemas" cognitivos de la realidad más ricos. Los "esquemas" son "estructuras preexistentes que dirigen la actividad perceptual y se modifican en su transcurso", son anticipaciones o planes de acción perceptual que tienen su origen en las experiencias pasadas, aunque la forma y organización de algunas de estas estructuras cognitivas (espacio, tiempo, idioma, etc.) 
está determinada genéticamente. Este concepto es la moderna versión del de "masa aperceptiva" de Herbart.

Neisser concibe la libertad como independencia y dominio de los demás por medio del conocimiento. El que posee "esquemas" cognitivos más ricos puede extraer más pronto y mayor información de la situación estimular y de una manera más objetiva. El perceptor que posee esquemas más ricos vive además menos "sometido a estímulo" y está mas "dirigido interiormente". Un buen ajedrecista extrae más y mejor información que uno malo, por poseer una mayor cantidad de "constelaciones esquemáticas". La diferencia entre ambos no es cuestión de verdad o falsedad, sino de ser capaz de observar más o menos cosas.

Según esta postura un hombre controla a otro, no mediante el condicionamiento, sino por el conocimiento ("esquemas") que posee de la información que el otro tiene. El jugador experto es difícil de vencer, porque posee más conocimientos y estrategias (información) que el contrincante.

La idea del hombre como computador de información es la predominante de esta escuela reciente de la psicología.

\section{EL MODELO COGNITIVO EMOCIONAL}

En 1983 H. Gardner en un interesante libro cuestionó seriamente el clásico modelo de la inteligencia racional, medida como "cociente de inteligencia" y expresada en términos de habilidades intelectuales. Este modelo dominó los campos del diagnóstico y selección de personal durante prácticamente este último siglo de psicología. A este modelo se le debe, en gran parte, el desarrollo de la moderna psicología científica, pero tuvo un fallo, que en la última década se está tratando de enmendar, el abandono del estudio del impacto de los procesos afectivo/emocionales sobre el comportamiento.

El origen del modelo de inteligencia racional está en la filosofía racionalista, pero, a nivel más operativo, habría que buscarlo en los descubrimientos psicométricos de Sir Francis Galton y su escuela (el concepto de percentil y de correlación). Un colaborador de Galton, Charles Spearman, publicó en 1904 dos interesantes trabajos que sirvieron de modelo a la psicometría y al análisis factorial de la inteligencia. Otros siguieron investigando en este campo y destacaron: Sir Cyril Burt en Londres, Godfrey H. Thompson en Edimburgo, y en Chicago, durante la década de 1930, Louis Leon Thurstone. Estos fueron los maestros de un gran número de psicólogos apasionados por el análisis factorial, que se encargaron de perfeccionar estadísticamente esta técnica y de inventar nuevas pruebas para medir los factores de inteligencia. 
En 1904, en Francia, Alfred Binet y Theodor Simon elaboraron la primera "escala métrica de la inteligencia" para comprobar el nivel intelectual de cada escolar en comparación con los otros niños de su edad. Las tres versiones que hubo de esta escala aparecieron en la revista Année Psychologique en 1905, 1908 Y 1911. La escala de Binet-Simon fue revisada y adaptada a la población norteamericana en la Universidad de Standford, primero por Lewis Terman (1916) y luego por M.A. Merril (1936). En 1911 W. Stern elabora el concepto de "cociente mental", posteriormente llamado por Terman "cociente intelectual", que sirve para expresar la capacidad intelectual, relacionando la "edad mental" del sujeto con su edad cronológica.

A partir de estos años muchas escalas y pruebas de inteligencia se elaboran y aplican masivamente para el diagnóstico y la de personal. Quienes obtenían la más selección alta calificación en estas pruebas eran consideradas las personas más aptas para los negocios y para conducir a los demás.

Pero ninguna de estas pruebas medía la capacidad emocional de las personas y sobre todo, como apuntó Gardner en su libro, ninguna medía la "inteligencia interpersonal", componente clave de la capacidad emocional y factor muy importante para el éxito profesional y para la madurez personal. De hecho, la experiencia está demostrando cada vez más -sobre todo en este momento histórico en el que el mercado de demanda se está imponiendo al viejo mercado de producción y de oferta- que se hace más necesaria la habilidad para las relaciones interpersonales y el propio control de las fuerzas emocionales.

El modelo de inteligencia emocional es propuesto claramente en 1990 por Peter Salovey, profesor de la Universidad de Yale, y John D. Mayer, profesor de la Universidad de Hampshire.

De hecho, quien más contribuyó a popularizar el concepto de inteligencia emocional fue el psicólogo norteamericano Daniel Goleman con su libro Inteligencia emocional (1994). Goleman define la inteligencia emocional como "la capacidad de motivamos a nosotros mismos, de perseverar en el empeño a pesar de las posibles frustraciones, de controlar los impulsos, de diferir las gratificaciones, de regular nuestros propios estados de ánimo, de evitar que la angustia interfiera con nuestras facultades racionales y, por último -pero no, por ello, menos importante- la capacidad de empatizar y confiar en los demás (Goleman, 1996, p.65). En todas y cada una de estas afirmaciones, desde la primera: "motivamos", hasta la última: "empatizar y confiar en los demás", está presente el "factor afectivo como modulador de la conducta.

Antonio R. Damasio, Director del Departamento de Neurología de la Facultad de Medicina de la Universidad de Iowa, publicó en el mismo año (1994) un interesante libro, El error de Descartes, que fundamenta sólidamente el proceso de la inteligencia emocional sobre las bases neuropsicológicas. 


\section{BIBLIOGRAFÍA BÁSICA}

\section{PARA EL MODELO CONDUCTISTA}

Skinner, B.F (1953). Science and Human Behavior. N. York: The Macmillan Co. (En castellano, Ciencia y conducta humana. Barcelona: Edil. Fontanella).

Skinner, B.F(1917). Beyond Freedom and Dignity. N. York: Alfred A. Knopf Publisher, (En castellano, Más allá de la libertad y la dignidad. Barcelona: Edit. Fontanella).

Skinner, B.F Cumulative Record: A Selection of Papers. N. York: Appleton-Century Crofts. (En castellano, Registro acumulativo. Barcelona: Fontanella).

Watson, J.B. Psichology as the Behaviorist Views it. Psychol. Rev., 193, 20, 158, 177.

Watson, J.B. The Place of the Conditioned Reflex in Psychology. Psychol. Rev. 23,89117.

Watson, J.B. (1925) Behaviorism. N. York:

Norton,. (En castellano, El conductismo. Buenos Aires: Edit. Paidos, 1961).

\section{PARA EL MODELO MATERIALISTA, DIALÉCTICO DE LA PSICOLOGÍA SOVIÉTICA}

Engels, F (1969) Ludwig Feuerbachy elfin de la filosofía clásica alemana. Madrid: Ricardo Aguilera Editor (original en 1886).

Engels, F. Introducción a la dialéctica de la naturaleza y El papel del trabajo en la transformación del mono en hombre. (Estos dos trabajos fueron escritos en 1873 y 1876 respectivamente). En castellano, Madrid: Edil. Ayuso, 1981.

Lenin, V.I. (1983). Materialismo y empiriocriticismo (En castellano. Moscú: Edit. Progreso) (original en 1908).

Lenin, V.I. (1983). Cuadernos filosóficos. (en castellano: Moscú: Edit. Progreso) (original 1914-1915).

Marx, C. y Engels, F (1974). La ideología alemana. (En castellano, Barcelona: Edit. Grijalbo) (original 1845-1846). 


\section{PARA EL MODELO HUMANISTA}

Allport, G. W. (1937). Personality: A Psychological Interpretacion. $N$ York: Holt. (En castellano, Psicología de la personalidad. Buenos Aires: Paidos, 1961).

Maslow, A.H. (1954). Motivation and Personality. N. York: Harper. (En castellano, Motivación y personalidad. Barcelona: Gráficas Sagitario, 1963).

Maslow, A.H (1962). Towards a Psychology of Being. Prnceton: D. van Nustrund. (En castellano, El hombre autorrealizado. Barcelona: Edit. Kairos, 1973).

Rogers, C.R. (1951). Client-CenteredTherapy; ita Current Practice, Implications and TIleory. Boston: Hoghton Miffin (En castellano, Psicoterapia centrada en el cliente. Buenos Aires: Paidos. 1969).

Rogers, C.R. (1961).On Becoming a Person Boston: Houghton Mifflin. (En castellano, el proceso de convertirse en persona. Mi técnica terapéutica. Buenos Aires: Paidos, 1972),

\section{PARA EL MODELO COGNITIVO}

Neisser, U. (1967). Cognitive psychology. N. York: Appleton-Century-Crofts. (En castellano, Psicología Cognitiva. México: Edit. Trillas, 1976).

Neissel; U. (1976). Cognition and Reality. Principles and Implications of Cognitivy Psychology. S. Francisco: W.H. Freeman and Co, (En castellano, Procesos cognitivos y realidad. Principios e implicaciones de la Psicología cognitiva. Madrid: Ediciones Marova, 1981).

Miller G.A., Galantel; E y Pribram, K.H. (1960). Plans and the Structure of Behavior. N. York: Holt, (En castellano, Planes y estructura de la conducta. Madrid: Edit. Debate).

\section{PARA EL MODELO COGNITIVO EMOCIONAL}

Ainsworth, M. D. S. y otros (1978). Patterns of Attachment. Hillsdale, New York: Earlbaum

Beck, A.T. (1976). Cognitive Therapy and the emotional Disorders. N. York: International! Universities Press. 
Damasio, A.R. (1996). El error de Descartes. La emoción, la razón y el cerebro humano. Barcelona: Grijalbo (original, 1994).

Ellis, A. (1980). Razón y emoción en psicoterapia. Bilbao: Desglée de Brower (original, 1962).

Gardner, H. (I983). Frames of Mind: The Theory 01 Multiple Intelligences. New York: Basic Books.

Goleman, Daniel (1996). Inteligencia Emocional. Barcelona: Kairos (original, 1995).

Rogers, CR. (1972). El proceso de convertirse en persona. Mi técnica terapéutica. Buenos Aires: Paidós (original, 1961).

Ribot, Th. (1905). La lógica de los sentimientos. Madrid: Daniel Sorro, editor. Publicación original en 1905.

Salovey, Peter y John D. Mayer (1990). "Emotional Intelligence" en Imagination, cognition and personality, 9, pp. 185-211.

Sutherland, S. (1992). Irrationality: The enemy within. Londres: Constable. Tversky, A. y D. Kahneman (1973). "Availabity: A heuristic for judging frecuency and probability". Cognitive Psychology, 2, pp. 207-232. 